\section{Analysis of the Auditory Feedback and Phonation in Normal Voices}

\author{
Mareike Arbeiter, MD', Simon Petermann, PhD', \\ Ulrich Hoppe, MD, PhD ${ }^{2}$, Christopher Bohr, MD', \\ Michael Doellinger, $\mathbf{P h D}^{\prime}$, and Anke Ziethe, $\mathbf{P h D}^{\prime}$
}

Annals of Otology, Rhinology \& Laryngology 2018, Vol. 127(2) 89-98

(c) The Author(s) 2017

Reprints and permissions:

sagepub.com/journalsPermissions.nav DOI: 10.1 1 77/0003489417744567

journals.sagepub.com/home/aor

(S)AGE

\begin{abstract}
Purpose: The aim of this study was to investigate the auditory feedback mechanisms and voice quality during phonation in response to a spontaneous pitch change in the auditory feedback. Does the pitch shift reflex (PSR) change voice pitch and voice quality? Quantitative and qualitative voice characteristics were analyzed during the PSR.

Method: Twenty-eight healthy subjects underwent transnasal high-speed video endoscopy (HSV) at 8000 fps during sustained phonation [a]. While phonating, the subjects heard their sound pitched up for 700 cents (interval of a fifth), lasting 300 milliseconds in their auditory feedback. The electroencephalography (EEG), acoustic voice signal, electroglottography (EGG), and high-speed-videoendoscopy (HSV) were analyzed to compare feedback mechanisms for the pitched and unpitched condition of the phonation paradigm statistically. Furthermore, quantitative and qualitative voice characteristics were analyzed. Results: The PSR was successfully detected within all signals of the experimental tools (EEG, EGG, acoustic voice signal, HSV). A significant increase of the perturbation measures and an increase of the values of the acoustic parameters during the PSR were observed, especially for the audio signal.

Conclusions: The auditory feedback mechanism seems not only to control for voice pitch but also for voice quality aspects.
\end{abstract}

\title{
Keywords
}

auditory feedback, phonation, pitch shift reflex, EEG, high-speed video endoscopy

\section{Introduction}

Auditory perception has been revealed to be one of the most important factors to control speech production. ${ }^{1-4}$ In particular, auditory feedback plays an important role in phonation in terms of stabilizing the fundamental voice frequency $\left(\mathrm{F}_{0}\right)$ while subjects compare the auditory feedback with the intended pitch and loudness and compensate mismatches in order to aim the target. ${ }^{2,5,6}$ The term pitch-shifted feedback was introduced by Burnett et $\mathrm{al}^{7}$ in experimental setups in that they presented an auditory feedback to the subjects that has been pitched up or down. When subjects perceived an auditory feedback that was higher than the intended pitch, they reacted with an increase in their $\mathrm{F}_{0}$. When subjects perceived an auditory feedback lower than the aimed pitch, they compensated by decreasing their $\mathrm{F}_{0}$. The physiological reaction of the adjustment of voice frequency in reaction to a perturbation of the auditory feedback is called the pitch shift reflex (PSR). With the PSR, the function of the auditory feedback that we need for corrections during phonation and speech can be investigated. Compensation in $\mathrm{F}_{0}$, in reaction to disturbances of the auditory feedback, was shown inter alia for sustained vocalization. ${ }^{8-11}$ Subjects reacted with a change of their $F_{0}$ with a latency of approximately 100 to 150 milliseconds ${ }^{7,8}$ and either with following or opposing frequency adjustments. ${ }^{8,9}$ The majority of responses during the investigations are opposing (for an increase in the frequency of the auditory feedback, the response would be a decrease in $\mathrm{F}_{0}$ and reverse) and seem to function as a compensatory mechanism. The responses were larger in amplitude for smaller stimuli than for larger stimuli for opposing responses. ${ }^{12}$

Further research found a correlation between the size of the stimulus (frequency difference) and the direction of the response. This has been shown in a previous study by Burnett et al, ${ }^{7}$ where it was found that when increasing the stimulus magnitude (in here from 25 to 300 cents), the percentage of opposing reactions decreases. ${ }^{8}$

\footnotetext{
'Department of Phoniatrics and Pediatric Audiology, ENT clinic, University hospital Erlangen, Medical school, Friedrich-AlexanderUniversity Erlangen-Nürnberg, Germany

${ }^{2}$ Department of Audiology, ENT clinic, University hospital Erlangen, Friedrich-Alexander-University Erlangen-Nürnberg, Germany
}

\section{Corresponding Author:}

Anke Ziethe, Universitatsklinikum Erlangen, Waldstr. Ib, Erlangen, 91054, Germany.

Email: anke.ziethe@uk-erlangen.de 
Thus, the direction of the response depends on the magnitude of the stimulus.

These findings lead to the assumption that small pitch perturbations are perceived as self-produced and counteractive responses might serve as a correcting mechanism for the stabilization of $\mathrm{F}_{0} \cdot{ }^{12,13}$ For the following responses, that were mainly observed for very large stimuli, for example, 500 cents, ${ }^{8}$ it was suggested that subjects try to adapt to their feedback as a reference. ${ }^{9}$

For longer lasting stimuli, with durations of 500 milliseconds and 1000 milliseconds, a second vocal response was also observed at approximately 300 milliseconds post stimulus. ${ }^{8,9}$ While the earlier vocal response seems to be more automatic and is mostly of an opposing (with regard to the stimulus) direction, the later response is often following. As this second response occurred with a longer latency post stimulus, a larger response magnitude with a higher accuracy, Hain et $\mathrm{al}^{9}$ assumed that this late response might be part of a more voluntary mechanism to consciously control voice frequency.

Beside the stimulus duration, other variations of the stimulus (eg, of loudness, pitch, and onset velocity) evoked modulations of the responses. Perturbation of voice loudness feedback leads to compensatory corrections in the voice amplitude. ${ }^{14}$ Larger response magnitudes were detected for pitch shift stimuli than loudness shift stimuli. ${ }^{15}$ With a generally higher $\mathrm{F}_{0}$, response magnitudes were larger and faster compared to a lower $\mathrm{F}_{0} \cdot{ }^{13}$ Moreover, larger vocal responses and shorter latencies were observed for experimental conditions with a higher fundamental frequency level compared to a lower one. ${ }^{16}$ When pitch shift velocity was decreased (from 1000 cents/sec to 330 cents/ $\mathrm{sec}$ ), subjects tended to react slower and less distinctly. ${ }^{15}$

The audio-vocal system seems not only to be flexible with regard to the nature of perturbation but also dependent of the sound type. Larger reactions occurred using real voices than using pure tones. In the presence of a mixed sound environment (voice mixed with nonvocal sounds), voice might be preferably changed in pitch prior to nonvoice sounds. ${ }^{11}$

Speech production is controlled by feedback and feedforward mechanisms involving auditory and somatosensory processes, located in the premotor cortex, primary motor cortex, and cerebellum. Feedback mechanisms compare the auditory input to a memorized model sound to verify if the sound matches with the intended sound production. Both mechanisms organize and execute control reactions to reach the desired target, but in contrast to the auditory feedback, it is the feedforward mechanism related to previously learned models and not to incoming sensory information and begins before the intended sound is produced. This is quantitatively and neuroanatomically described as the DIVA model (Directions Into Velocities of Articulators). ${ }^{17,18}$ The DIVA model was supported by further gathered neuroimaging functional magnetic resonance imaging(fMRI) data that showed bilateral activation of the superior temporal cortex during pitch shift experiments. Since activity in the right frontal cortex has also been seen, it was suggested that neurone in the superior temporal lobe that work as detecting error cells communicate to pitch-correcting motor cells in the prefrontal cortex. Experimental conditions with no perception of an error in auditory feedback revealed neuronal activity in the left frontal cortex. Perturbation in the auditory feedback leads to right hemispheric participation for correction processes. ${ }^{19}$

Currently, the influence of the perturbation in the auditory feedback and the influence of the stimulus on latency, magnitude, and direction of the voice response are known and linked with neurophysiological results. In summary, previous studies investigated primarily the quantitative aspects of the physiological voice response. A study of Leydon et $\mathrm{al}^{20}$ supports the assumption that auditory feedback is also important to fine-tune the voice and thus possibly voice quality. In their study, vibratolike oscillations of the voice in $\mathrm{F}_{0}$ were maintained by the control loop of the auditory feedback. That study focuses on the qualitative aspects of the phonation during the PSR. We aim to investigate if the auditory feedback mechanism only controls for voice pitch or if the voice quality will also be adapted. This is an important aspect for voice therapy. If patients with a functional voice disorder do not have a sufficient working auditory feedback process, the adaptation of the voice quality can be affected. We therefore hypothesize for normal voices that the PSR can be evoked in all participants and that the timing and auditory control is in normal range compared with previous studies. Furthermore, we suggest that due to the meshing auditory and kinaesthetic control mechanisms, the adaptation of the pitch and an adaptation of the voice quality take place.

\section{Aims}

In the present study, the authors examine the auditory feedback processes, the physiological voice response, and the voice quality during the PSR during sustained phonation. For this purpose, the pitch shift reflex is provoked in healthy adults. The PSR is used due to the larger magnitudes of the physiological reaction on the individual's own voice signal than on pure tone feedback.

In the present study, high-speed-videoendoscopy (HSV) is used as an additional tool to visually detect the PSR and analyze vocal fold dynamics of the phonation. This technique enables the recording of the real-time vocal fold dynamics and is especially suitable for the analysis of qualitative laryngeal characteristics during pitch changes and thus during the PSR. ${ }^{21}$

The PSR will be investigated via electroencephalography (EEG), the acoustic voice signal, electroglottography 


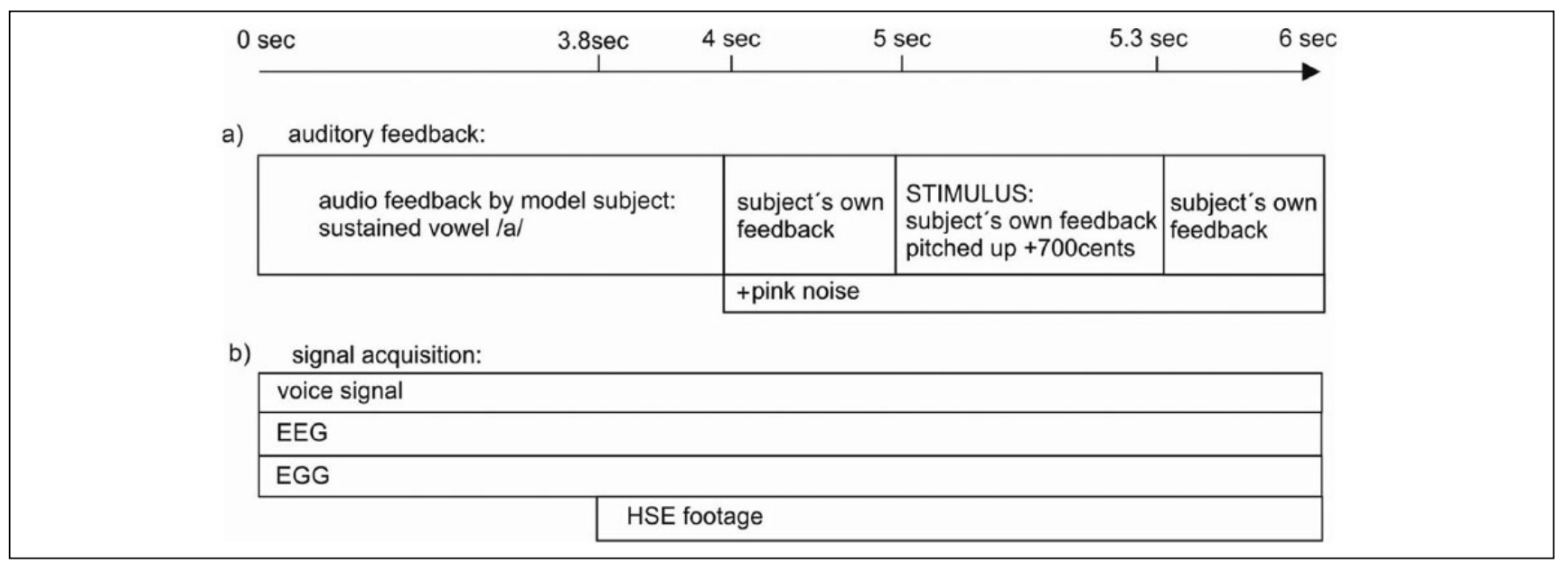

Figure I. (a) Auditory feedback with pitch-shifted feedback between 5.0 and 5.3 seconds. (b) Acquisitions of the 4 signals: voice, electroencephalography (EEG), electroglottography (EGG), and high-speed-videoendoscopy (HSV) footage.

(EGG), and the high-speed video signal. Transnasal highspeed videoendoscopy will be applied to gather additional information on the physiological processes underlying the PSR by analyzing vocal fold dynamics synchronously to the other signals.

\section{Material and Methods}

\section{Participants}

Twenty-eight participants ( 7 male, 21 female; age range: 20-30 years) volunteered in this study. All subjects were native German speakers with no history of neurological deficits or speech or voice disorder, and none of them were trained singers. All participants passed a hearing screening at $20 \mathrm{~dB}$ SPL. All participants signed a consent form. The study confirms the principles of the declaration of Helsinki and was approved by the Ethics Committee of the University hospital Erlangen (No. 4364).

\section{Procedures}

The detailed experimental setup was already introduced in Petermann et $\mathrm{al}^{22}$ and will be explained only briefly for the main understanding. During the first 4 seconds of the experiment, the subjects heard a model voice phonating a sustained vowel /a/ and had to imitate it for 6 seconds, regardless of any auditory disturbance they might hear (see Figure 1). The subjects were not enlightened about the purpose of the experiment. After presentation of the model voice, the subjects heard their own recorded voice for $1 \mathrm{sec}-$ ond, which was afterwards pitch-shifted by 700 cents (interval of a fifth) for 300 milliseconds. For the remaining 700 milliseconds, the pitch-shifted feedback turned back again into the subjects' own feedback (Figure 1). The unit cents is used to compare music intervals or sound pitches with each other. It is correlated with the hearing sense, and thus 100 cents is 1 equal staged note $\left(i=\log _{2} p\right.$ Oktave). The conversion from 700 cents into a music interval is about an interval of a fifth.

Pink noise with an upper cutoff frequency of $900 \mathrm{~Hz}$ supplied the background to reduce bone-conducted feedback. For female subjects, a female model voice with a frequency at $220 \mathrm{~Hz}$ was used and for male subjects a male model voice at $110 \mathrm{~Hz}$.

Each subject repeated the phonation paradigm 20 times. The auditory feedback was played at $75 \mathrm{~dB}$ SPL, and the subjects maintained a sound pressure level between 75 and $85 \mathrm{~dB}$ SPL. For analysis of the pitch shift reflex, the signals of the EEG, EGG, acoustic voice signal, and high-speed video signal were recorded. The experiment took place in a sound-attenuating and electrically shielded chamber, and the subjects wore a Logitech Premium Stereo Headset (USB port, $22.05 \mathrm{kHz}$ ) including a microphone for the presentation of auditory feedback and recording the subject's voice.

Additionally, all subjects wore a necklace with 2 EGG electrodes. Furthermore, EEG electrodes were placed across the subjects' scalp. During the whole experiment, a transnasal flexible endoscope attached to a high-speed camera recorded the vocal fold vibrations (Figure 2a).

Four signals were acquired: EEG to detect the N100 as a neurophysiological correlate for the PSR, acoustic voice signal, EGG, and HSV footage for monitoring the vocal fold vibration. The first 3 signals were acquired during the whole experiment for 6 seconds. The HSV footage was recorded from second 3.68 until 5.8 (Figure 1). To reduce the amount of the recorded data, this interval was chosen as the most important time span to capture the physiological response of the pitch shift reflex. 


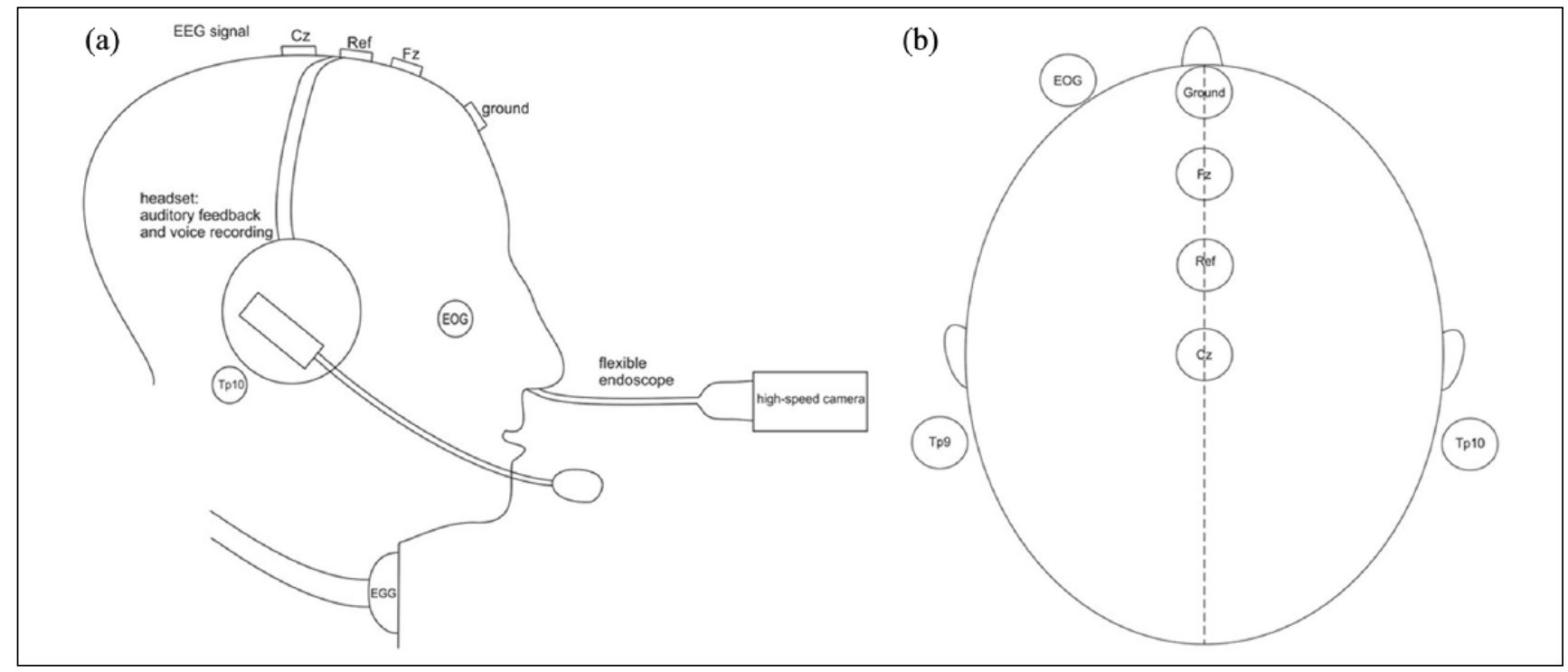

Figure 2. (a) Experimental setup: subject with electroencephalography (EEG) electrodes, headset, flexible endoscope, with highspeed camera. (b) The EEG electrodes following the 10-20 system.

\section{Apparatus/Data Acquisition}

In addition to the acoustic voice signal, the EEG, EGG, and video signal were acquired as follows.

EEG. A setup of 7 EEG electrodes was applied to measure the event-related potentials (ERPs) following the 10 to 20 system. ${ }^{23}$ Six electrodes were distributed across the subject's scalp (Figure 2b).

The main electrodes $\mathrm{Cz}$ and $\mathrm{Fz}$ were placed on the scalp, in between them Fcz serving as a reference, plus a ground electrode placed on the forehead. Furthermore, 1 electrode was placed on the mastoid on each side (Tp9 and Tp10) and 1 electrode below the left eye to detect artifacts by eye movement (electrooculography). The impedance of each electrode was below $5 \mu \mathrm{V}$. After gathering the data, the EEG was referenced from Fcz to the mean of the mastoid electrodes (Tp9 and $\mathrm{Tp} 10)$. Recordings were made using a BrainAmp amplifier (Brain Products GmbH, Gilching, Germany) connected to the PC via a Brain Products adapter box and the Brain Vision recorder software 2.0 (Brain Product).The EGG was recorded via the Laryngograph Processor (Laryngograph Ltd, Wallington, England).

High-speed videoendoscopy. Vocal fold oscillations were recorded by a transnasal laryngoscope with fiber optics (Olympus ENF GP, Olympus GmbH, Hamburg, Germany) that was coupled to a Photron SA1.1 (Photron Ltd, Tokyo, Japan) high-speed camera. The camera was installed on an extension arm and connected to the flexible laryngoscope via a $25-\mathrm{mm}$ objective (Stortz, Tuttlingen, Germany). We recorded the vocal fold oscillations with $8000 \mathrm{fps}$ at a spatial resolution of $128 \times 128$ pixel. The complete experimental setup is shown in Figure 3.

\section{Data Processing}

EEG. For the measurement of the neuronal response to the pitch perturbation, the detection of the N100 was chosen. The N100 was interpreted as the time of perception of the deviant stimulus, namely, the PSR. Therefore, the EEG recording of an interval that reaches from 100 milliseconds before stimulus onset until 500 milliseconds after stimulus onset was used for the analysis. The EEG data with less than $-100 \mu \mathrm{V}$ or more than $100 \mu \mathrm{V}$ were excluded from further analysis as they were categorized as nonphysiological reactions. For every subject, a grand average was computed over all experimental trials and both main electrodes, $\mathrm{Fz}$ and $\mathrm{Cz}$. The most prominent negative peak in an interval from 80 milliseconds to 150 milliseconds after stimulus onset was detected automatically as the N100 by using Matlab. $^{24}$

Acoustic and EGG signal. The computation of the fundamental frequency for all subjects was performed in Praat (Amsterdam, Netherland). For each subject, the $\mathrm{F}_{0}$ of the 500-millisecond interval before stimulus onset was compared to the 500-millisecond interval after stimulus onset using a Matlab script. None of the data were normally distributed, and the Wilcoxon signed rank test was used. The statistical tests were performed in intervals of 1 millisecond.

The most prominent peak of statistically significant intervals, lasting at least 20 milliseconds, was determined as the PSR. 


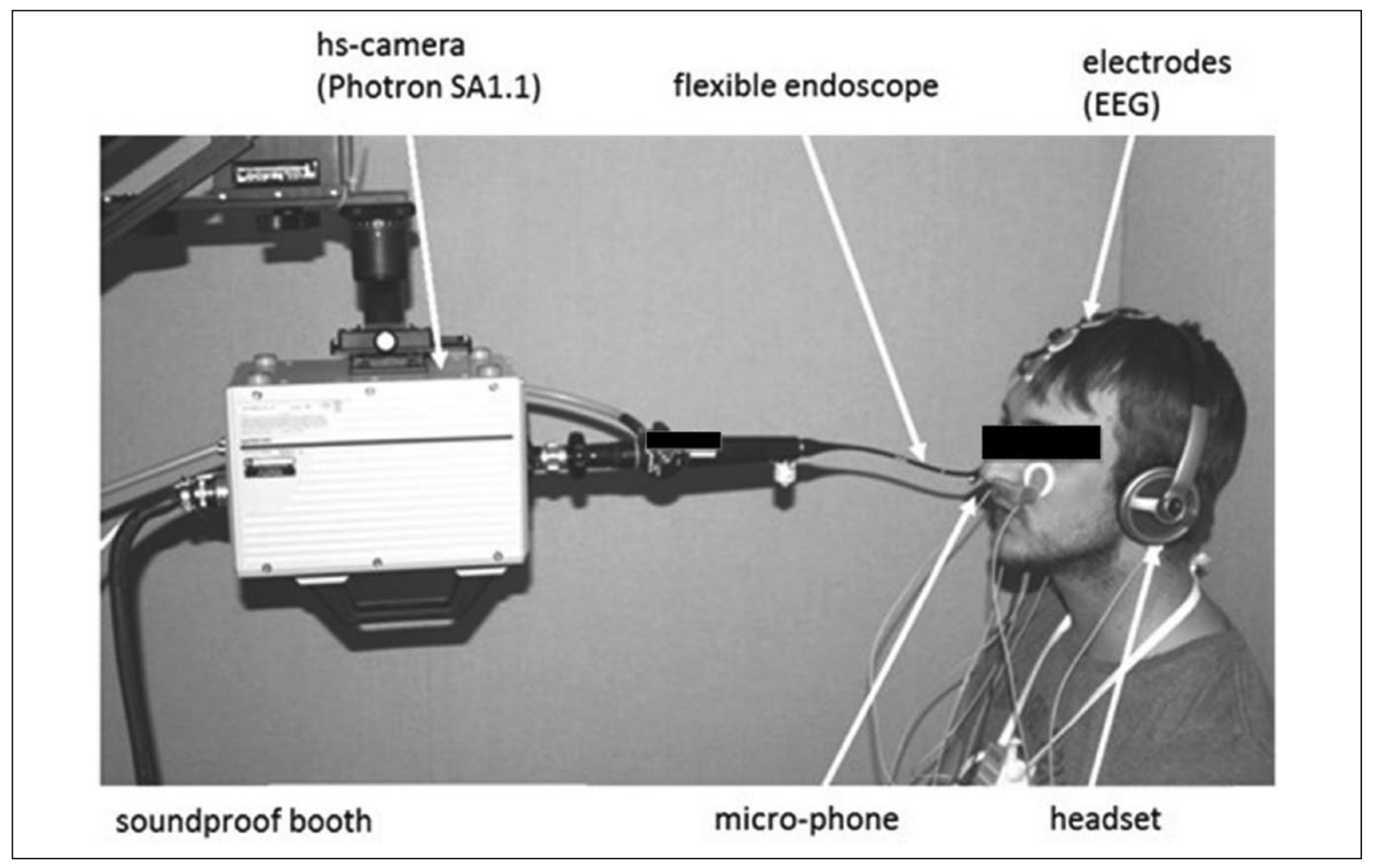

Figure 3. Experimental setup with high-speed-videoendoscopy, electroencephalography, and electroglottography for the measurement of the pitch shift reflex within the phonation paradigm.

High-speed videoendoscopy. The recorded high-speed videos were segmented, ${ }^{25}$ which means the glottal area was marked and hence detected. The glottal area waveform (GAW), which is the function of glottis pixels over time (see Figure 4 ), was computed and the $\mathrm{F}_{0}$ extracted.

\section{Data Analysis}

To detect the PSR for each subject, the fundamental frequency of the 500-millisecond interval before stimulus onset was compared to the fundamental frequency of the 500-millisecond interval after stimulus onset. Each interval of 500 milliseconds was partitioned into 23 subintervals, on which the statistical tests were performed. Every subinterval lasted 60 milliseconds, with an overlap of 40 milliseconds to the next subinterval. Voice pitch responses to a stimulus are expected at least 60 milliseconds post stimulus. $^{7}$

For the acoustic voice signal, EGG, and high-speed video, the fundamental frequency, perturbation, and acoustic measures were analyzed via the Glottis Analysis Tools (GAT), which was developed at the division of Phoniatrics and Pediatric Audiology at the university hospital, Erlangen, Germany, to investigate the vocal fold movement and hence

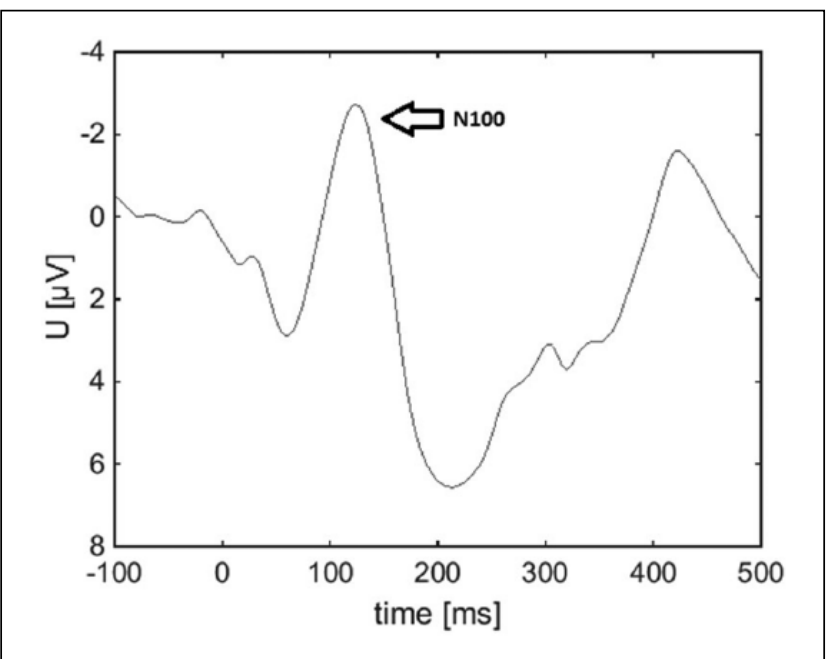

Figure 4. Grand average of electroencephalography measurements over all subjects with $\mathrm{NIO0}$ at I23 millisecondss. The curve is reflected with respect to the $\mathrm{x}$-axis.

the voice quality during the PSR. The parameters were extracted for a time span of 500 milliseconds before and 500 milliseconds after stimulus onset. Each interval of each 
Table I. Analyzed Perturbation Measures and Voice Quality Parameters.

\begin{tabular}{|c|c|}
\hline HNR (harmonics to noise-ratio) & Yumoto et al. $1982^{26}$ \\
\hline NNE (normalized noise energy) & Kasuya et al. $1986^{27}$ \\
\hline Snrv-I (signal to noise ratio) & Klingholz $1990^{28}$ \\
\hline CPP (cepstral peak prominence) & Hillenbrand et al. $1994^{29}$ \\
\hline Spectral flatness & Lessing $2007^{30}$ \\
\hline Shimmer & Bielamowicz et al. $1996^{3}$ \\
\hline $\begin{array}{l}\text { APQ-3 (amplitude perturbation } \\
\text { quotient) }\end{array}$ & $\begin{array}{l}\text { Michaelis 1999; Lessing } \\
2007^{32,30}\end{array}$ \\
\hline APF (amplitude perturbation factor) & $\begin{array}{l}\text { Michaelis 1999; Lessing } \\
2007^{32,30}\end{array}$ \\
\hline Jitter & $\begin{array}{l}\text { Bielamowicz et al. 1996; } \\
\text { Inwald et al. 201 }{ }^{11,33}\end{array}$ \\
\hline PPQ (pitch perturbation quotient) & $\begin{array}{l}\text { Michaelis 1999; Lessing } \\
2007^{32,30}\end{array}$ \\
\hline
\end{tabular}

parameter of the prestimulus period was compared to its corresponding poststimulus match. The acquired parameters were in voice assessment commonly used acoustic and perturbation measures for the objective description of voice quality: fundamental frequency, harmonics-to-noise-ratio (HNR), normalized noise energy (NNE), signal to noise ratio (SNR-v1), cepstral peak prominence (CPP), spectral flatness, shimmer, amplitude perturbation quotient (APQ-3), amplitude perturbation factor (APF), jitter, and PPQ-3 (see Table 1). The perturbation measures jitter and shimmer and their derivatives APQ-3 (shimmer computed over 3 cycles), APF (measure of period perturbation), and PPQ-3 (jitter computed over 3 cycles) were analyzed for the fluctuations in period and amplitude of the vocal fold oscillations. Harmonics to noise ratio gives the ratio between the harmonic and the nonharmonic parts of the voice signal. Similarly, the SNR displays the portion of noise in the acoustic voice signal. Together, HNR, SNR, spectral flatness, and NNE estimate the noise level in voiced speech signals and are used within the present study to describe voice quality within normal voices. The periodicity of the voice signal respectively the breathiness can be also determined with the CPP. An aperiodic voice signal results in decreased CPP.

\section{Statistics}

The statistics were computed in SPSS Statistics 21 (2012; IBM Corp, Armonk, New York, USA) and MatLab. All responses of the subjects were checked for normal distribution for every period and parameter using the KolmogorovSmirnov test. The Wilcoxon signed rank test was used for all data (not normally distributed) to analyze significant differences between the physiological responses before (unpitched condition) and after (pitched condition) stimulus onset. More precisely, every parameter was checked for statistically significant differences between before and after the stimulus onset. For all statistical tests, the level of significance was $P<.05$.

\section{Results}

\section{EEG}

Out of all valid physiological responses (562 trials), the grand average was computed. The maximum peak was found at 123 \pm 18 milliseconds after stimulus onset with an amplitude of $-4.3 \mu \mathrm{V}$. The grand average waveform is shown in Figure 4.

\section{Acoustic Voice Signal}

Altogether, 17 subjects (5 male, 12 female) showed a PSR, and 11 subjects did not show any statistically significant voice pitch response. For all subjects (see Table 2), a grand average waveform of the acoustic voice signal was computed. For this waveform of the acoustic voice signal, the maximum peak of the PSR was detected at $338 \pm 139$ milliseconds with a maximum pitch change of $10.6 \pm 5.8$ cents (see Table 2, Figure 5).

\section{Audio/Video/EGG Analysis}

The authors defined a successful PSR detection if a statistically significant change in the fundamental frequency in the reference signal (voice response), the acoustic voice signal, could be detected.

In all 3 signals (acoustic voice signal, EGG, and video [glottal area waveform]), a PSR could be observed descriptively for 17 subjects (see Figure 5) but without statistical validation (see Table 3). Corresponding to our definition of the PSR, the statistically significant intervals of the acoustic voice signal were further analyzed.

For all 17 subjects, a statistically significant change of the fundamental frequency in the audio signal was found for the intervals 6 (100-160 milliseconds) and 9 to 11 (160-260 milliseconds), ranging from 100 milliseconds to 260 milliseconds post stimulus $(6: p=.03 ; 9: p=.005 ; 10: p=.039 ; 11: p=$ $.023)$. These 4 intervals, with an occurring PSR, were further analyzed regarding the acoustic and perturbation parameters.

\section{Acoustic Voice Signal}

For all 4 intervals, the acoustic and perturbation parameters of all signals were compared between the "pitched" condition (prestimulus) and "no pitch" condition (poststimulus). The perturbation parameter Shimmer\% $(9: P=.047)$ and the acoustic parameters CPP $(6: p=.001 ; 9: p=.009 ; 10$ : $p=.001), \operatorname{HNR}(6: p=.025)$, and spectral flatness $(6$ : $p=.001 ; 9: p=.006 ; 10: p=.04)$ of the audio signal changed significantly between pre- and poststimulus (see Table 3 ). Spectral flatness was significantly changing in the EGG signal between the interval before and within the pitch shift $(11: P=.007)$. For the video signal, Shimmer\% $\%$ (6: $p=.003$; 9: $p=.043)$ and the $\operatorname{SNR}(6: p=.02)$ could be detected as changing parameters during the PSR. 
Table 2. Results of Subjects Showing a Voice Pitch Response in the Phonation Paradigm.

\begin{tabular}{lcccc}
\hline & $V P_{L}$ (Milliseconds) & $V P_{M}$ (Cents) & $N 0_{L}$ (Milliseconds) & $N 100_{M}(\mu V)$ \\
\hline Subjects (mean) & $338 \pm 139$ & $10.6 \pm 5.8$ & $123 \pm 18$ & $-4.3 \pm 3.4$ \\
\hline
\end{tabular}

Abbreviations: $\mathrm{NIO0}_{\mathrm{L}}$, latency of the $\mathrm{NI00} ; \mathrm{NIO0}_{\mathrm{M}}$, magnitude of the $\mathrm{NIO0} ; \mathrm{VP}_{\mathrm{L}}$, latency of the voice pitch response; $\mathrm{VP}_{\mathrm{M}}$, magnitude of the voice pitch response.

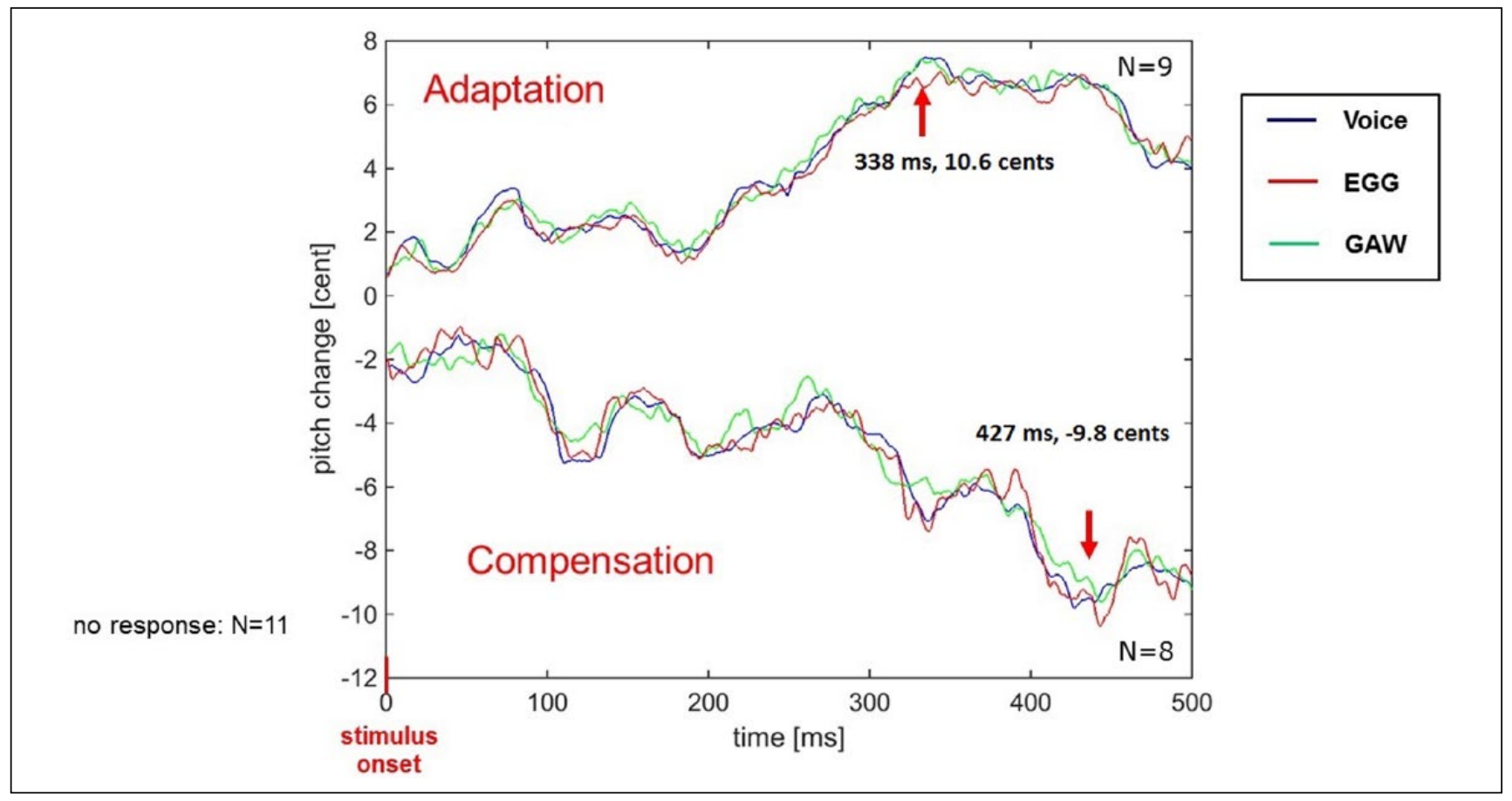

Figure 5. Grand average of the electroencephalography signal, acoustic voice, and video signal showing time of the pitch shift reflex and the pitch change of all subjects.

\section{Discussion}

The aim of this study was to detect the pitch shift reflex as a neurophysiological response to a pitch perturbation in the auditory feedback by the analysis of the EEG, acoustic voice signal, the EGG signal. Furthermore, we wanted to investigate if during the PSR changes in the quality of phonation occur. Therefore, we added high-speed video endoscopy to visualize vocal fold dynamics during the pitch shift response. The PSR was evoked by pitching up the subjects' own voice by 700 cents for 300 milliseconds during the sustained phonation of the vowel $/ \mathrm{a} /$.

\section{$E E G / N 100$}

A grand average waveform of the EEG was computed over all subjects and resulted in the N100 with a peak at 123 milliseconds and amplitude of $-4.3 \mu \mathrm{V}$. Similar latencies of 123 milliseconds \pm 13 milliseconds and 129 milliseconds \pm 4 milliseconds ${ }^{34}$ were found in previous studies, partly for 200 milliseconds lasting stimuli pitched up by 200 cents. $^{24}$
The results are in range with the reported latencies of related studies, and therefore, it can be concluded that the perception of the PSR could be detected in the EEG of all subjects.

\section{Acoustic Voice Signal: Response Latency and Magnitude}

For response latency and magnitude, all subjects show a high intra- and intervariability (Table 2). These findings correspond with previous studies: Burnett et $\mathrm{al}^{8}$ found pitch latencies ranging from less than 100 milliseconds to more than 400 milliseconds after stimulus onset, and Liu et $\mathrm{a}^{16}$ observed latencies of less than 100 milliseconds to more than 300 milliseconds after stimulus onset. ${ }^{35}$ Other studies reported values between 200 and 300 milliseconds $^{14,36}$ and also shorter latencies as 130 milliseconds $^{9}$ and 106 milliseconds. ${ }^{11}$ Most studies used the same duration of stimuli presentation as it was used in the presented study (300 milliseconds) but with a smaller magnitude of the stimulus 
Table 3. Significant Intervals (PSR) and Parameters for all Subjects. ${ }^{a}$

\begin{tabular}{|c|c|c|c|c|c|c|}
\hline Interval & freq & HNR & Snrvl & CPP & spectrF & Shim \\
\hline \multicolumn{7}{|c|}{6 (I00-I60 milliseconds) } \\
\hline Audio & .03 & .025 & & .001 & .001 & \\
\hline Video & & & .02 & & & .003 \\
\hline \multicolumn{7}{|c|}{ EGG } \\
\hline \multicolumn{7}{|c|}{9 (I60-220 milliseconds) } \\
\hline Audio & .005 & & & .003 & .006 & .047 \\
\hline Video & .030 & & & & & .043 \\
\hline \multicolumn{7}{|c|}{ EGG } \\
\hline \multicolumn{7}{|c|}{10 (I80-240 milliseconds) } \\
\hline Audio & .039 & & & .003 & .040 & \\
\hline Video & .030 & & & & & \\
\hline \multicolumn{7}{|l|}{ EGG } \\
\hline \multicolumn{7}{|c|}{ II (200-260 milliseconds) } \\
\hline Audio & .023 & & & & .007 & \\
\hline Video & .012 & & & & & \\
\hline EGG & & & & & & \\
\hline
\end{tabular}

${ }^{a} P$ values are given. CPP, cepstral peak prominence; freq, frequency; HNR, harmonics to noise ratio; Shim, shimmer; snrvI, signal-to-noise ratio VI; spectrF, spectral flatness.

of 100 cents (vs. 700 cents). Hence, we conclude that the stimulus magnitude does not seem to affect response latency.

Likewise, the averaged response magnitudes for all subjects in this study are about 10.6 cents, ranging from 4.19 to 20.93 cents, that is comparable with the magnitudes found in previous studies. For a physiological voice response, evoked by a pitch-shifted feedback of 100 cents, multiple authors reported varying response magnitudes of -7.27 cents, ${ }^{24} 69$ cents, ${ }^{9}-13.1$ cents, ${ }^{36} 20$ cents, ${ }^{37}$ and 22 cents. ${ }^{11}$ A smaller pitch shift stimulus of 50 cents provoked even an equal response magnitude of 18.5 cents. $^{36}$ Considering previous research studies, the stimulus magnitude of 700 cents led to a rather small response magnitude compared to the stimulus magnitudes of 100 and 50 cents. It seems that a larger stimulus magnitude does not necessarily lead to a larger response magnitude but to a rather smaller response. Furthermore, the large stimulus could have evoked none response in 11 out of 28 subjects. Normally, during phonation and speech, subjects rely on their auditory feedback for error detection. Regarding the nonresponders, they could also have relied on their kinesthetic feedback.

\section{Acoustic Parameters and Perturbation Measures}

The perturbation parameter shimmer of the acoustic voice signal and video signal could be pointed out as significantly changing parameter during the PSR. These findings confirm the fact that a perturbation in auditory feedback leads to modifications of $\mathrm{F}_{0}$, causing less perturbation in form of fluctuations in period and amplitude in the acoustic voice signal and also in the GAW of the video signal, reflecting the fundamental frequency. In conclusion, these findings display fewer irregularities of vocal fold vibrations during the PSR (Video: Shim\%: pre: $14.6 \% \pm 10.6$, PSR: $13.8 \% \pm 11.3)$. Regarding the acoustic parameters, especially the HNR, SNR v1, and the CPP, and spectral flatness alter significantly between both conditions (no pitch vs pitch). Analyzing the referred parameters during the PSR, it is obvious that the SNR increases, the spectral flatness decreases and that the values of CPP fluctuate, showing a reduced noise level and thus reduced breathiness in the voice. These findings confirm the assumption that the PSR provokes a voice quality-enhancing process. However, the HNR in the audio signal decreases and displays also limitations within the adaptation process of the PSR regarding quality enhancement. These results support the assumption of a fine-tuning of the voice during phonation that facilitates more intentional and accurate laryngeal settings resulting in a better voice quality. The auditory feedback process can therefore be described as control mechanism for quantitative (pitch) and possibly for qualitative aspects (voice quality) of phonation. Consequently, in case of an insufficient working auditory feedback process, the adaptation of the voice and voice quality can be affected. Patients with functional voice disorders (muscle tension dysphonia, MTD) often show problems with their self-perception during voice therapy. Possibly, these are related to an insufficient working auditory feedback. Therefore, it can be important to monitor and train auditory feedback processes in patients with voice disorders. Further investigations with patients with MTD should be conducted to clarify these assumptions/hypotheses. 


\section{Conclusion}

The PSR could successfully be detected with a similar latency and magnitude of the physiological response compared to previous research results. Regarding the voice quality during the PSR, the analysis of the voice quality parameters of the high-speed video and the EGG signal indicates the PSR as a physiological process for possible voice quality enhancement. Consequently, the auditory feedback process controls for voice pitch (quantitative aspect) and presumably also for quality. Thus, auditory feedback should also be monitored and trained in case of a (persisting) voice disorder.

\section{Acknowledgements}

The present work was performed in fulfilment of the requirements for obtaining the degree 'Dr med.' at the Friedrich-AlexanderUniversity Erlangen-Nürnberg.

\section{Declaration of Conflicting Interests}

The author(s) declared no potential conflicts of interest with respect to the research, authorship, and/or publication of this article.

\section{Funding}

The author(s) disclosed receipt of the following financial support for the research, authorship, and/or publication of this article: This work was supported by the German research foundation (DFG) under grant no. DO1247/4-1.

\section{References}

1. Fairbanks G. Selective vocal effects of delayed auditory feedback. J Speech Hear Disord. 1995;20(4):333-346.

2. Siegel GM, Pick HL. Auditory feedback in the regulation of voice. J Acoust Soc Am. 1974;56(5):1618-1624.

3. Binnie CA, Daniloff RG, Buckingham HW. Phonetic disintegration in a five-year-old following sudden hearing loss. $J$ Speech Hear Disord. 1982;47(2):181-189.

4. Siegel GM, Kennard KL. Lombard and sidetone amplification effects in normal and misarticulating children. $J$ Speech Hear Res. 1984;27(1):56-62.

5. Fairbanks G. Systematic research in experimental phonetics. I. A theory of the speech mechanism as a servosystem. $J$ Speech Hear Disord. 1954;19(2):133-139.

6. Howell P, Powell DJ. Delayed auditory feedback with delayed sounds varying in duration. Percept Psychophys. 1987;42(2):166-172.

7. Burnett TA, Senner JE, Larson CR. Voice F0 responses to pitch-shifted auditory feedback: a preliminary study. $J$ Voice. 1997;11(2):202-211.

8. Burnett TA, Freedland MB, Larson CR, Hain TC. Voice F0 responses to manipulations in pitch feedback. $J$ Acoust Soc Am. 1998;103(6):3153-3161.

9. Hain TC, Burnett TA, Kiran S, Larson CR, Singh S, Kenney MK. Instructing subjects to make a voluntary response reveals the presence of two components to the audio-vocal reflex. Exp Brain Res. 2000;130(2):133-141.
10. Jones JA, Munhall KG. Perceptual calibration of F0 production: evidence from feedback perturbation. J Acoust Soc Am. 2000;108(3 Pt 1):1246-1251.

11. Sivasankar M, Bauer JJ, Babu T, Larson CR. Voice responses to changes in pitch of voice or tone auditory feedback. $J$ Acoust Soc Am. 2005;117(2):850-857.

12. Behroozmand R, Korzyukov O, Sattler L, Larson CR. Opposing and following vocal responses to pitch-shifted auditory feedback: evidence for different mechanisms of voice pitch control. J Acoust Soc Am. 2012;132(4):2468-2477.

13. Larson CR, Sun J, Hain TC. Effects of simultaneous perturbations of voice pitch and loudness feedback on voice F0 and amplitude control. J Acoust Soc Am. 2007;121(5 Pt1): 2862-2872.

14. Bauer JJ, Mittal J, Larson CR, Hain TC. Vocal responses to unanticipated perturbations in voice loudness feedback: an automatic mechanism for stabilizing voice amplitude. $J$ Acoust Soc Am. 2006;119(4):2363-2371.

15. Hain TC, Burnett TA, Larson CR, Kiran S. Effects of delayed auditory feedback (DAF) on the pitch-shift reflex. $J$ Acoust Soc Am. 2001;109(5 Pt 1):2146-2152.

16. Liu H, Auger J, Larson CR. Voice fundamental frequency modulates vocal response to pitch perturbations during English speech. J Acoust Soc Am. 2010;127(1):EL1-EL5.

17. Guenther FH, Hampson M, Johnson D. A theoretical investigation of reference frames for the planning of speech movements. Psychol Rev. 1998;105(4):611-633.

18. Guenther FH. Cortical interactions underlying the production of speech sounds. J Commun Disord. 2006;39(5):350-365.

19. Tourville JA, Reilly KJ, Guenther FH. Neural mechanisms underlying auditory feedback control of speech. Neuroimage. 2008;39(3):1429-1443.

20. Leydon C, Bauer JJ, Larson C. The role of auditory feedback in sustaining vocal vibrato. J Acoust Soc Am. 2003;114(3): $1575-1581$

21. Hoppe U, Rosanowski F, Döllinger M, Lohscheller J, Schuster M, Eysholdt U. Glissando: laryngeal motorics and acoustics. J Voice. 2003;17(3):370-376.

22. Petermann S, Döllinger M, Kniesburges S, Ziethe A. Analysis method for the neurological and physiological processes underlying the pitch-shift reflex. Acta Acust United Acust. 2016;102:284-297.

23. Klem GH, Lüders HO, Jasper HH, Elger C. The tentwenty electrode system of the International Federation. Electroencephalogr Clin Neurophysiol Suppl. 1999;52: 3-6.

24. Behroozmand R, Korzyukov O, Larson CR. Effects of voice harmonic complexity on ERP responses to pitch-shifted auditory feedback. Clin Neurophysiol. 2011;122(12):2408-2417.

25. Lohscheller J, Toy H, Rosanowski F, Eysholdt U, Döllinger M. Clinically evaluated procedure for the reconstruction of vocal fold vibrations from endoscopic digital high-speed videos. Med Image Anal. 2007;11(4):400-413.

26. Yumoto E, Gould WJ, Baer T. Harmonics-to-noise ratio as an index of the degree of hoarseness. J Acoust. Soc. Am. 1982; 71(6):1544-9.

27. Kasuya H, Ogawa S, Mashima K, Ebihara S. Normalized noise energy as an acoustic measure to evaluate pathologic voice. J Acoust Soc Am. 1986;80(5):1329-34. 
28. Klingholz F. The measurement of the signal-to-noise ratio in continouus speech. Speech Communication 1987;6(1):15-26.

29. Hillenbrand J, Cleveland RA, Erickson RL. Acoustic correlates of breathy vocal quality. J Speech Lang Hear Res. 1994;37(4):769-778.

30. Lessing J. Entwicklung einer Klassifikationsmethode zur akustischen Analyse fortlaufender Sprache unterschiedlicher Stimmgüte mittels Neuronaler Netze und deren Anwendung [Development of a classification method for the acoustic analysis of continuous speech of different vocal quality by means of neural networks their applications]. Göttingen, Germany: Georg-August-Universität Göttingen, MathematischNaturwissenschaftlich Fakultät, 2007.

31. Bielamowicz S, Kreiman J, Gerratt BR, Dauer MS, Berke GS. Comparison of voice analysis systems for perturbation measurement. J Speech Hear Res. 1996;39:126-134.

32. Michaelis D. Das Göttinger Heiserkeits-Diagramm -Entwicklung und Prüfung eines akustischen Verfahrens zurobjektiven Stimmgütebeurteilung pathologischer Stimmen [Development and testing of an acoustic method for objective voice quality assessment of pathological voices].
Dissertation zur Erlangung des Doktorgrades, MathematischNaturwissenschaftliche Fakultät, Georg-August-Universität Göttingen, 1999.

33. Inwald EC, Döllinger M, Schuster M, Eysholdt U, Bohr C. Multiparametric analysis of vocal fold vibrations in healthy and disordered voices in high-speed imaging. $J$ Voice. 2011;25:576-590.

34. Li W, Chen Z, Liu P, Zhang B, Huang D, Liu H. Neurophysiological evidence of differential mechanisms involved in producing opposing and following responses to altered auditory feedback. Clin Neurophysiol. 2013;124(11):2161-2171.

35. Liu H, Larson CR. Effects of perturbation magnitude and voice F0 level on the pitch-shift reflex. J Acoust Soc Am. 2007;122(6):3671-3677.

36. Liu P, Chen Z, Jones JA, Huang D, Liu H. Auditory feedback control of vocal pitch during sustained vocalization: a crosssectional study of adult aging. PloS One. 2011;6(7):e22791.

37. Larson $\mathrm{CR}$, Altman $\mathrm{KW}$, Liu H, Hain TC. Interactions between auditory and somatosensory feedback for voice F0 control. Exp Brain Res. 2008;187(4):613-621. 